\title{
A Combinatory-Algebraic Perspective on Multipartiteness, Entanglement and Quantum Localization
}

\author{
Ioannis Raptis*and Romàn R. Zapatrin ${ }^{\dagger}$
}

\begin{abstract}
We claim that both multipartiteness and localization of subsystems of compound quantum systems are of an essentially relative nature crucially depending on the set of operationalistically available states. In a more general setting, to capture the relativity and variability of our structures with respect to the observation means, sheaves of algebras may need be introduced. We provide the general formalism based on algebras which exhibits the relativity of multipartiteness and localization.
\end{abstract}

\section{Prolegomena cum Physical Motivation}

The non-local behavior of quantum systems is virtually undisputed. There is ample experimental evidence suggesting that there exist quantum states of an essentially non-local nature, an issue which is verified by the statistics of observations. Entanglement is a crucial resource for quantum information processing and quantum communication. As it turns out, while it may be easy to produce non-entangled states, it is difficult to fabricate and maintain entangled ones. Recently, multipartite entanglement has been classified by the use of partitions of the set of subsystems [1].

We may regard this as the first indication of the idea we wish to explore below, namely, the relativity of the 'property' for a subsystem to be observed -in effect, to be localized-somewhere. Albeit, it is perhaps inadequate to just say relativity; one should also say uncertainty of some sort, as the position itself is created at the very moment of the preparation of the state. Quanta act not only non-locally, but also ' $a$-locally' 2, 4], as if there is no given external physical space, fixed up-front as it were, to restrain their 'quantum leaps of coherence and entanglement'. Even more iconoclastically, space(time) is intuited to be 'inherent' in quanta, as it were, created by them [5]. On the whole, it

*EU Marie Curie Fellow, Theoretical Physics Group, Blackett Laboratory, Imperial College of Science, Technology and Medicine, London SW7 2BZ, UK; e-mail: i.raptis@ic.ac.uk

${ }^{\dagger}$ Quantum Information Group, ISI, Villa Gualino, V.le S.Severo 65, 10133, Torino, Italy; e-mail: zapatrin@isiosf.isi.it (address for correspondence) 
is more physical to maintain that space(time) and its mathematical analysis (topology and geometry) is a result of the algebraically represented (dynamical) relations between quanta rather than being fixed up-front, once and forever, by the theorist. We may distill all this to the following motto:

First comes the quantum, then space; not the other way around.

Organization of the paper. The paper is organized as follows. We begin with an overview of how multipartiteness arises in classical mechanics, what are the ways to recover it operationally, and to what extent it is 'absolute'. We point out that in the case of the lack of availability of all states, it may turn out that even classical systems may exhibit the 'virtual' character of their multipartite structure. This is just an observation from classical statistics. Then we provide the necessary basic definitions and recall how multipartiteness is described in standard quantum mechanics. We observe that the relativity of localization and entanglement already exists in the usual quantum mechanics, so that there is no need to add to or to remove from the standard theory essentially anything.

We then translate both classical and quantum multipartite issues into a uniform algebraic language. This enables us to introduce the notion of multipartite structures (MPS) on algebras in a way that crucially depends on the set of available states. Then we show how the structure of loci of subsystems - which we claim to be the very structure of space(time) - emerges rather naturally. At the end, we entertain the possibility that observation-relativization and, concomitantly, locus-variability may be mathematically modelled by (finitary) sheaves of (Rota, in known cases [4]) algebras over those loci-structures (spectral topologies) inherent in quantum subsystems much in the same way that has been accomplished for quantum spacetime foam [5] and gravity [3].

\section{Classical compoundness}

In this section we describe two ways in which the compoundness of classical systems may be treated as relative. We commence the study of compoundness starting from classical systems. We show that even at the classical level there are essentially two different manifestations of the relativity of the notion of multipartiteness. The first manifestation is due to coarse-graining (grouping subsystems), while the second is related to different decompositions of the available configuration space, decompositions which depend on the scope of sets an experimenter possesses at her disposal (ie, available or 'experimentally accessible' states). In this way we introduce the twofold relativity of the notion of compoundness: compoundness based on coarse-graining, and compoundness based on the choice of available states, which we treat as being uncorrelated.

Cartesian product structures. How can we actually verify that a (classical) system is indeed multipartite? Let us consider a simple model. On the one hand we have a classical system whose configuration space $S$ consists of 9 points, while 
on the other, two classical systems each having a 3-point configuration space, say $M_{1}$ and $M_{2}$. The 'first level' mathematical description of them is identical: the configuration space consists of 9 points, be it $S$ or $M_{1} \times M_{2}$. We are not able yet to tell whether the first system is 'here' and the second one is 'there' solely in terms of their configuration space description. A Cartesian Product Structure (CPS) must be imposed in order to draw such distinctions.

How can one impose a CPS on a set? A way to put it is by hand. In our example this looks like making a rectangle from a line-see Fig. 1.

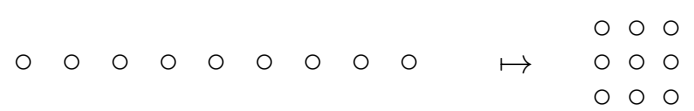

Figure 1: An illustration of how CPS is imposed.

Coarse-graining. How many CPSs can one introduce on a given finite set of cardinality $n$ ? An immediate answer is the following: each possible CPS is associated with a particular factorization $n=n_{1} \cdot n_{2} \cdots n_{k}$, where $k$ indicates the number of subsystems associated with this particular CPS. Then, up to permutations of the factors, all CPSs are in 1-1 correspondence with the factorizations of the number $n$.

Given a certain factorization $n=n_{1} \cdot n_{2} \cdots n_{k}$, we can consider groups of factors as factors. In other words, we may not 'exhaust' the factorization, as it were, carry it to its 'finest' or 'irreducible' level-ie, to $n$ 's prime factors. Thus, instead of an 'ultra-fine' description of subsystems, we consider coarser ones. This is the well known notion of coarse-graining [6, 9]

However, this approach is too rigid; in particular, it entails that configuration spaces with a prime number of points have no CPSs at all, and obviously the same holds in the quantum case, see section 3 .

Compound systems with constraints. Our first step towards a realistic description of multipartiteness is to take into consideration that the states and the observables of a physical system may have different 'accessibility status'. In particular, some of them may turn out to be unavailable in our experimental setting. When we have a configuration space equipped with CPS, the stateswhich are probability distributions - may be product or not.

Now, instead of declaring up-front a CPS on a set, let us try to go the other way around and consider the case when two classical parties are far away from each other so that it takes considerable effort to make their states correlated. That means, we distinguish between states which are 'easy' for us to prepare and those which are not. The formulation of the inverse problem beckons: given a configuration space $S$ and a collection of states, how can one recover a CPS $S=M_{1} \times M_{2}$ such that the available states are product or weakly correlated ones? 
This is a purely statistical problem which can be solved by using principal component analysis. The idea is that the distributions are viewed as points in a multi-dimensional space and then an eigenvalue problem is solved, so that the number of resulting vectors with sufficiently large eigenvalues gives the number of principal factors, which in our case may be viewed as the number of subsystems. What is swept out by cutting 'sufficiently small' eigenvalues are correlated states which, according to our setting of the problem, are rare and pragmatically inaccessible.

Suppose that we have a 'really' bipartite classical system, but one which also has constraints. This means that there are states in the Cartesian product configuration space which are not accessible for us. Therefore, the 'effective configuration space' will no longer carry a Cartesian product structure; rather, it will be its proper subset.

Our claim is the following. CPSs can be introduced irrespectively of the extent to which we can decompose the number of points of the available configuration space. The only relevant issue is the analysis of correlations. Therefore, from now on we change the spelling of ' $\mathrm{C}$ ' in CPS from 'Cartesian' to 'classical' and CPSs become CLASSICAL PRODUCT STRUCTURES.

Constrained systems viewed algebraically. Now, let us base our considerations on an algebraic ground. Suppose we have a bipartite system, and we measure local observables $A_{1}$ and $A_{2}$. If the state $\rho$ in which we measure them is a product one, the mean value of the product equals the product of the values:

$$
\rho\left(A_{1} \cdot A_{2}\right)=\rho\left(A_{1}\right) \cdot \rho\left(A_{2}\right)
$$

This may be regarded as a characteristic property. If we have two algebras $\mathfrak{a}_{1}$ and $\mathfrak{a}_{2}$ of local observables, then for any product state and any $A_{1} \in \mathfrak{a}_{1}$, $A_{2} \in \mathfrak{a}_{2}$, condition (11) holds.

Our idea is to forget for the time being about the CPS on the configuration space and start from a given set $P$ of states which we declare to be product. Then we may take two subalgebras $\mathfrak{a}_{1}$ and $\mathfrak{a}_{2}$, and ask whether for any $\rho \in P$ (11) holds. If the answer is yes, then operationally, from the point of view of available observations, we are dealing with a bipartite system. Note that this approach is perfectly applicable to systems with constraints; when the effective configuration space has no product structure, the multiplicativity (1) still holds!

Note that when we have a CPS on a set, we can consider local algebras of observables. In turn, each local algebra has its set of points. What is the relation between the global algebra of observables and local ones, between the overall configuration space and the configuration spaces of the individual systems? The answer is known. The global algebra is (in general, a superset of) the tensor product of local algebras, and the overall configuration space is (in general, a superset of) the Cartesian product of individual configuration spaces.

This gives us a clue to introduce multipartiteness in an algebraic fashion. Namely, take a collection of subalgebras of the overall algebra of observables. For any state $\rho$ we may ask if the analog of (1) holds. Note that this is not 
an attempt to treat states as points, which is meaningless, since for two local observables (11) does not hold in general. That is why we introduce the term loci instead of points.

Classical product structures. In classical mechanics the algebra of observables is a (commutative) algebra of smooth functions defined on the configuration space. Due to Gel'fand duality we may generalize the ideas of section 2 and introduce CPS in a purely algebraic way, that is, with no a priori reference to the underlying geometrical configuration space (manifold), which only later will be recovered by the representation theory of the algebras employed much in the same way we did for spacetime foam in [5. A Classical Product Structure (CPS) $\mathfrak{P}$ is a set of unital subalgebras.

$$
\mathfrak{P}=\left\{\mathfrak{a}_{1}, \ldots, \mathfrak{a}_{n}\right\}
$$

The algebras $\mathfrak{a}_{i}$ forming this set are said to be ALGEBRAS OF LOCAL OBSERVABLES. The labels which mark each subalgebra is called LOCUS. This definition looks at first sight counterintuitive as it apparently disagrees with the standard viewpoint: (i) we do not require the local subalgebras to intersect only at the unit element of the embracing algebra and the reason for this is because they may be indistinguishable from the unit element when we have a limited number of states at our disposal. (ii) we do not require the local subalgebras to comprise the whole algebra of observables, this too reflecting our 'local experimental ignorance' concerning the totality of properties of the quantum system that can in principle be observed.

State-CPS duality. Given an algebra $\mathfrak{A}$, a CPS $\mathfrak{P}=\left\{\mathfrak{a}_{1}, \ldots, \mathfrak{a}_{n}\right\}$ and a state $\rho$ on it, we say that $\rho$ is PRODUCT WITH RESPECT TO $\mathfrak{P}$ whenever the generalization of the condition (11) holds:

$$
\forall i \quad \forall A_{i} \in \mathfrak{a}_{i} \quad \rho\left(\prod_{i} A_{i}\right)=\prod_{i} \rho\left(A_{i}\right)
$$

Therefore we may encounter the following situation. Suppose we have a set of states, which we may regard as being 'easily available'. Then, it may happen that there are several inequivalent CPSs with respect to which these states are product.

Our claim is the following. Even in the classical case, in a situation where we have a restricted set of states at our disposal, all product structures can be treated as full fledged multipartite(ness) as we have no operational means to single out, 'prefer', or discriminate between particular states. Now we may approach the basic claims of our paper. Even in a classical situation the following hold. What creates observable multipartiteness? The set of available states. Where is the multipartite structure genuinely imposed? On the algebra of observables. 


\section{Compoundness in quantum mechanics}

In this section we show that all the issues concerning the relativity of classical multipartiteness are still effective in the quantum case. Furthermore, the variety of quantum multipartite structures acquires a new, continuous degree of freedom. The main difference between the classical and the quantum case is that the condition for a state to be product is replaced by separability.

The relativity of multipartite entanglement. Begin with basic definitions. Given a state of a composite $N$-partite system $\mathbf{S}$, denote its density matrix by $\rho$. A density matrix $\rho$ is called PRODUCT if it can be represented as a tensor product of density matrices of subsystems $\rho=\rho_{1} \otimes \ldots \otimes \rho_{N}$. A state $\rho$ is SEPARABLE if its density matrix is a convex or 'incoherent' (ie, a classical probabilistic) linear combination of product ones.

$$
\rho=\sum p_{\alpha} \rho_{1}^{\alpha} \otimes \ldots \otimes \rho_{N}^{\alpha}
$$

with $p_{\alpha} \geq 0$ and $\sum p_{\alpha}=1$.

The states which are not separable are called ENTANGLED.

The first hint we wish to give towards introducing our algebraic picture of compoundness is based on the following rationale: when we have several parties in an entangled state we must consider them as a single party - an inseparable entity. At the same time, what right have we got to still call this 'coherent whole', 'several entangled parties'? Presumably, because in principle we also have at our disposal other states, which are separable and, a fortiori, which can separate or individuate the constituent parties.

Coarse-graining. To introduce the quantum version of coarse-graining in CPS we proceed in close analogy with the classical case, the only, albeit essential, difference being that the aforementioned separability condition should also be taken into account.

So, let us weaken the condition for states of a composite system $\mathbf{S}$ to be product and separable. Namely, instead of requiring it to be product with respect to $\mathcal{H}=\mathcal{H}_{1} \otimes \cdots \otimes \mathcal{H}_{N}$, we make this condition relative to a partition $\Sigma$ of the set $\mathbf{A}$ of subsystems of $\mathbf{S}$, that is, with respect to a particular decomposition of the set of subsystems. In a sense, we are allowed to relax in this way the product and separable states of a composite system, because, as explained earlier, they are precisely the 'least quantum' ones (ie, non-entangled).

Given a partition $\Sigma=\left\{\sigma_{1}, \ldots, \sigma_{M}\right\}$ of $\mathbf{A}$ and a density matrix $\rho$ in the state space of $\mathbf{S}, \rho$ is called $\Sigma$-PRODUCT whenever it can be represented as a tensor product $\rho=\rho_{\sigma_{1}} \otimes \cdots \otimes \rho_{\sigma_{M}}$ and $\Sigma$-SEPARABLE if it is a convex combination of $\Sigma$-product states:

$$
\rho=\sum p_{\alpha} \rho_{\sigma_{1}}^{\alpha} \otimes \ldots \otimes \rho_{\sigma_{N}}^{\alpha}
$$


In other words, (4) means that we can prepare $\rho$ as an ensemble of mixed states located at sites $\sigma_{1}, \ldots, \sigma_{M}$.

Given a state $\rho$, we may now ask for each partition $\Sigma$ of the set $\mathbf{A}$ of subsystems of $\mathbf{S}$ whether $\rho$ is $\Sigma$-separable or not. As a result, we obtain the set $\Pi(\rho)$ of partitions of $\mathbf{A}$ with respect to which $\rho$ is separable (4):

$$
\Sigma \in \Pi(\rho) \quad \Leftrightarrow \quad \rho \quad \text { is } \Sigma \text {-separable }
$$

The set of all partitions of a given set has a natural ordering ' $\preceq$ ', which represents acts of coarse-graining those partitions. In order to specify all partitions with respect to which a given state $\rho$ is separable, we only have to find maximal ones with respect to ' $\preceq$ '. This may serve a base for geometrico-algebraic invariants for multipartite entanglement, for details of which the reader may refer to [10].

Tensor product structures (TPSs). In Section 2 we introduced the variety of CPS for classical systems. This admits an immediate generalization to quantum systems which was carried out by Svozil [7] and Zanardi [8]. Let us briefly review it. First, following [7], take an arbitrary basis in a Hilbert space $\mathcal{H}$ of dimension $n$ with no a priori given tensor product structure. Take any factorization $n=n_{1} \cdots n_{k}$ and associate with it $k$ partitions of the set of basis vectors. The partitions must be such that each basis vector could be represented as an intersection of appropriate elements of each partition. In other words, these partitions should form independent Boolean algebras. Then, taking an element of a partition, we may view it as a qubit (in generalized sense with an arbitrary number of values). To get the next degree of freedom in producing different TPSs, apply according to [8] a global unitary transformation $U: \mathcal{H} \rightarrow \mathcal{H}$, which will yield us isomorphic, but different (with respect to, say, observables), qubit structures. In the sequel, the subsystems of the associated multi-party decomposition will be referred to as virtual.

So, by now we have completed the description of both classical and quantum multipartite systems. What we have done was essentially to show how given a compound system we can describe it in different ways and in some sense 'modify' its compoundness. The main goal of our paper is, however, to provide the appropriate algebraic machinery for creating compoundness. We address this issue in the next section.

\section{Compoundness from an algebraic perspective}

As it has been pointed out above, both classical and quantum compound systems exhibit some kind of relativity of their multipartite structure. In this section we take a step further and turn the construction the other way around. Starting from a given set of observables and states - in fact, this is perhaps the only way that our approach may qualify as being operationalistic proper-we create rather than (re)construct (and this is our main claim here!) the full fledged multipartite structure of compound quantum systems. It is full fledged, because 
no experiment can be devised to discriminate between our 'fake' multipartiteness and the purported 'real' one. As a matter of fact, we abide to the stronger statement that there is simply no 'real' multipartiteness at all.

We shall describe both classical and quantum systems by using algebraic means. That is, we shall regard 'algebras of observables' as primary theoretical objects. As a result, the geometrical configuration and state spaces will turn out to be just representation spaces for those algebras. This is in line with a generalized notion of Gel'fand duality [5]. Note that the term 'algebra of observables' is rather broadly and heuristically used here, as only its self-adjoint elements correspond to observables proper. The algebra itself is broader as it embodies both observables and evolutions. Recall the basic definitions.

Now we are going to provide a quantum, noncommutative analog of the classical product structures. The language of algebras is so adequate for it that we do not have to introduce practically anything new. The main difference between the classical and the quantum case is that the variety of MPS in the latter becomes much broader and richer than in its classical counterpart.

Virtual multipartite structures. A collection $\mathfrak{P}$

$$
\mathfrak{P}=\left\{\mathfrak{a}_{i}\right\}_{i \in \operatorname{Loc}(\mathfrak{P})}
$$

of unital subalgebras of $\mathfrak{A}$ which are closed with respect to taking double commutant $\forall i\left(\mathfrak{a}_{i}\right)^{c c}=\mathfrak{a}_{i}$ is called VIRTUAL MULTIPARTITE STRUCTURE (MPS).

There is a partial order on MPS which enables us to represent the possibility of coarse-graining in both classical and quantum MPSs. It is introduced by analogy with that on partitions. Namely, we say that an MPSs $\mathfrak{P}=\left\{\mathfrak{a}_{i}\right\}_{i \in \operatorname{Loc}(\mathfrak{P})}$ is COARSER than $\mathfrak{P}^{\prime}=\left\{\mathfrak{a}_{i}\right\}_{i \in \operatorname{Loc}(\mathfrak{P})}\left(\right.$ denote it by $\left.\mathfrak{P} \preceq \mathfrak{P}^{\prime}\right)$ if we can partition $\mathfrak{P}^{\prime}$ so that the span of each element of the partition is a subalgebra of appropriate $\mathfrak{a}_{i}$ from $\mathfrak{P}$.

Furthermore, this is a lattice ordering. In fact, if we have a set of MPSs $\mathfrak{P}^{1}, \ldots, \mathfrak{P}^{r}$ we may take all possible intersections of all subalgebras from all MPPSs. The result will be again an MPS which will be the least upper bound of $\mathfrak{P}^{1}, \ldots, \mathfrak{P}^{r}$ with respect to the relation " $\preceq "$

$$
\mathfrak{P}^{1} \vee \ldots \vee \mathfrak{P}^{r}=\bigcap_{i=1}^{r} \bigcap_{k^{i} \in \operatorname{Loc}\left(\mathfrak{P}^{i}\right)}\left\{\mathfrak{a}_{k^{i}}^{i}\right\}
$$

The lattice structure can then be used to recover the loci.

The state-MPS duality. We emphasize that we did not require the elements of different 'local' subalgebras to commute. This for instance is in striking contrast to the usual (involutive) observable algebras of (relativistic) quantum matter systems which are already localized in Minkowski space, as the geometry of the fixed background spacetime dictates the 'commutativity vis-à-vis local causality' properties of the corresponding algebras (Einstein Locality). Here, exactly because we do not posit up-front an ambient base localization 
space(time), we are not a priori constrained by Einstein Locality and, as a result, quantum non-locality effects do not surprise us. This is the crucial point of our approach - we shall require commutativity only on certain states which we treat as being 'available'. Operationally, that means that the values of all 'local' variables should be independent random (stochastic) variables, but under the proviso that the system is in an available state only. We thus define the relation $\mathfrak{S}$ of separability between a state $\rho$ and an MPS $\mathfrak{P}$ as follows

$$
\rho \mathfrak{S P} \quad \Leftrightarrow \quad \forall a_{i} \in \mathfrak{a}_{i} \forall a_{j} \in \mathfrak{a}_{j} \rho\left(\mathfrak{a}_{i} \cdot \mathfrak{a}_{j}\right)=\rho\left(\mathfrak{a}_{i}\right) \cdot \rho\left(\mathfrak{a}_{j}\right)
$$

Note that the rhs of $(\sqrt{7})$ is well defined as both $\mathfrak{a}_{i}, \mathfrak{a}_{j}$ belong to the total algebra $\mathfrak{A}$. The analog of local observables in standard tensor stutures are the operators of the form $1 \otimes \cdots \otimes A_{i} \otimes \cdots \otimes \mathbf{1}$. Then, by analogy with (5) we introduce the duality using the same notation $\Pi(\rho)$ for the appropriate set of MPSs:

$$
\mathfrak{P} \in \Pi(\rho) \quad \Leftrightarrow \quad \rho \quad \text { is } \mathfrak{P} \text {-separable }
$$

Recovering the loci. As claimed above, we start with an algebra $\mathfrak{A}$ of observables and the set $\mathfrak{S}$ of available states. With any state $\rho \in \mathfrak{S}$ we can associate the set $\Pi(\rho)$ of MPSs with respect to which $\rho$ is separable.

The loci of any of MPS from $\Pi(\rho)$ are still thought of as groups of elementary subsets. Now, using the lattice structure on the set of all MPSs, we can form the supremum

$$
\mathfrak{P}_{\mathfrak{S}}=\bigvee_{\rho \in \mathfrak{S}} \bigvee_{\mathfrak{P} \in \Pi(\rho)} \mathfrak{P}
$$

which is still an MPS. This is exactly what provides us with 'points' - namely, the loci $\operatorname{Loc}\left(\mathfrak{P}_{a} v l s t\right)$ are treated as ultimately indivisible with respect to the given set of accessible states, which in turn one may identify with the available (microscopic) energies.

How and why does relativity come about? When we broaden the range of available states (as it were, increase the energy of microscopic resolution) the number of terms in (9) may only increase, therefore the MPPSs $\mathfrak{P}_{\mathfrak{S}}$ may become finer. This in turn means that its loci 'decay' or break down to 'smaller' ones. The benefit we get from this construction from the point of view of quantum computing is that when the loci are defined (created) we can then directly apply Svozil's [7] partition scheme in order to reconstruct qubits. These are fully fledged qubits viewed purely operationally.

We conclude the paper by noting briefly that in the already worked out case where the 'compound' system is quantum spacetime, the aforesaid algebras have been seen to be (non-involutive) Rota algebras, while the set of loci was endowed with a so-called spectral Rota topology by employing a variant of the idea of Gel'fand duality coined Gel'fand spatialization [4, 5]. This theoretical scenario is supposed to represent a combinatory-algebraic description of (the kinematics of) spacetime foam - the conception of the spacetime microtopology as being a quantum observable [5]. At the same time, it has been intuited 
that, by further regarding these algebras as noncommutative local rings, their sheaf-theoretic localizations over their Gel'fand spectra can capture, by entirely algebraico-categorical means, notions of relativity and dynamical variability of quantum discretized spacetime [3].

Thus, similarly here we intuit that the structure of loci may be endowed with a suitable spectral topology and, concomitantly, their relativity and dynamical variability can be captured by considering sheaves of the relevant associative algebras over these topological spaces. Such raw analogies may provide the fertile ground for future investigations in which ideas from quantum computation proper can be brought closer to ones from quantum spacetime and gravity.

\section{References}

[1] Dür, W., and Cirac, J.-I., Physical Review A, 61, 042314 (2000)

[2] Finkelstein, D. R., Quantum Relativity: A Synthesis of the Ideas of Einstein and Heisenberg, Springer-Verlag, Berlin-Heidelberg-New York (1996)

[3] Mallios, A. and Raptis, I., Finitary Spacetime Sheaves of Quantum Causal Sets: Curving Quantum Causality, International Journal of Theoretical Physics, 40, 1885 (2001); gr-qc/0102097

[4] Raptis, I. and Zapatrin, R. R., Quantization of discretized spacetimes and the correspondence principle, Int. J. Theor. Phys., 39, 1 (2000); grqc/9904079

[5] Raptis, I. and Zapatrin, R. R., Algebraic description of spacetime foam, Classical and Quantum Gravity, 20, 4187 (2001); gr-qc/0102048

[6] Sorkin, R., Finitary Substitute for Continuous Topology, International Journal of Theoretical Physics, 30, 923 (1991)

[7] Svozil, K., Quantum information in base n defined by state partitions, Physical Review Letters, A666, 044306 (2002); quant-ph/0105031

[8] Zanardi, P., Virtual Quantum Subsystems , Physical Review Letters, 87, 077901 (2001); quant-ph/0103030

[9] Zapatrin, R. R., Pre-Regge Calculus: Topology Via Logic, International Journal of Theoretical Physics, 32, 779, (1993)

[10] Zapatrin, R. R., Combinatorial Topology Of Multipartite Entangled States,, Journal of Modern Optics (2002), to appear; eprint quant-ph/0207058 